\title{
Avances en el tratamiento de la tuberculosis multirresistente
}

\author{
CARLOS PEÑA M.*y VICTORINO FARGA C.**
}

\section{Advances in the treatment of multi-drug resistant tuberculosis}

Therapy of multi-drug resistant tuberculosis (MDR TB) is based on trials with drugs with highly variable patterns of resistance and non-standardized follow-ups that make it difficult to provide recommendations with strong levels of evidence. Also, the vast majority of MDR-TB patients fail to receive therapy and those who receive it, only achieve around $50 \%$ of good results. Fortunately new drugs have emerged (bedaquiline, delamanid, pretomanid) that are being useful for these patients with better designed trials and monitoring, in which the efficacy, toxicity and degree of relapses can be evaluated more accurately. Some drugs already known (fluorquinolones, linezolid and clofazimine) are also being introduced in new schemes of therapy.

Key words: Tuberculosis, multidrug-resistant; bedaquiline; oxazoles.

\section{Resumen}

El tratamiento de las tuberculosis multidrogorresistentes (TBC-MDR) se basa en esquemas de fármacos con diseños muy variables, en pacientes con patrones de resistencia heterogéneos y seguimientos no estandarizados, lo que hace dificil plantear recomendaciones con fuerte nivel de evidencia. Además, sólo una minoría de estos enfermos recibe tratamiento a nivel mundial y con los actuales esquemas menos del 50\% de los que logran ser tratados curan. Afortunadamente, durante los últimos años han aparecido nuevos medicamentos, (bedaquilina, delamanid y pretomanid), que están demostrando ser de real utilidad para estos pacientes en ensayos con mejor diseño y seguimiento, donde se puede establecer con mayor precisión la eficacia, toxicidad y grado de recaídas. Además, algunos fármacos ya conocidos, (fluoroquinolonas, linezolid, clofazimina) están siendo introducidos dentro de los nuevos esquemas de tratamiento.

Palabras clave: Tuberculosis multidrogorresistente; bedaquilina; oxazoles.

\section{Introducción}

La TBC-MDR (resistente a isoniacida y rifampicina) y la TBC-XDR (resistente además a fluoroquinolonas y drogas inyectables de segunda línea) son consideradas actualmente un grave problema de salud pública a nivel mundial. Las TBC multirresistentes son una señal de mal manejo de los programas de control de la tuberculosis, con fallas en la supervisión del tratamiento inicial de los enfermos. La prevención del desarrollo de resistencia bacteriana, por malos tratamientos, es esencial para evitar el desarrollo de resistencia bacteriana. Se predice que habrá un dramático aumento en estas distintas formas de TBC multirresistente en las próximas décadas en todo el mundo y que los casos derivarán cada vez con más frecuencia de transmisiones persona a persona que de enfermos mal tratados. Las terapias tradicionales de las TBC multirresistentes son caras, tóxicas y muy prolongadas, lo que atenta contra su eficacia y la adherencia a los tratamientos. El esquema convencional de la Organización Mundial de la Salud (OMS), de

\footnotetext{
* Encargado Programa de Control y Eliminación de la Tuberculosis (PROCET), Servicio de Salud Metropolitano Central.

** Asesor del Ministerio de Salud.
} 
20 meses de duración, tiene una fase intensiva de 8 meses diarios con kanamicina, pirazinamida, ofloxacino, protionamida y cicloserina, continuando por 12 meses más con ofloxacino, protionamida y cicloserina, administrados también diariamente $^{1}$. Este esquema tiene un bajo índice de curación (30-50\%), lo que implica que una importante proporción de estos pacientes continúa transmitiendo la enfermedad en la comunidad ${ }^{2-4}$. Adicionalmente, hasta 9 a $15 \%$ de los casos que fracasan contribuyen a la amplificación de la resistencia a otros fármacos ${ }^{5-7}$.

En el año 2014 la OMS estimó en 9 millones los nuevos casos de TBC en el mundo, de los cuales alrededor de 480.000 (5,3\%), corresponden a TBC-MDR; de ellos, 43.000 (9\%), son además, TBC-XDR ${ }^{8}$. Los resultados de curación y la letalidad para la TBC-MDR son 50\% y $16 \%$ respectivamente y para la TBC-XDR, $26 \%$ y $30 \%$. Estos antecedentes han motivado una intensa área de investigación clínica tendiente a encontrar soluciones terapéuticas mejores que las disponibles actualmente. Afortunadamente, han aparecido esquemas acortados a 9-12 meses, como el de Bangladesh, de menor duración y mayor eficacia (84 a 87,9\%) el que ya es recomendado por la OMS para el tratamiento de las TBC-MDR ${ }^{9-10}$.

En los últimos años se han introducido nuevos fármacos, como bedaquilina, delamanid y pretomanid, para la terapia de TBC-MDR y TBC-XDR, pero aún no se genera un suficiente conocimiento sobre su eficacia y toxicidad. Además, se han agregado otros agentes ya conocidos, como fluoroquinolonas, linezolid y clofazimina, para reforzar los nuevos esquemas terapéuticos. Los estudios con diversas combinaciones de estos medicamentos presentan problemas en su diseño y metodología, siendo muy complejos de interpretar, de alto costo y de larga duración, sin estandarización de los objetivos y con falta de consensos y coordinación entre los distintos investigadores. Estos obstáculos dificultan establecer políticas globales para el control de la TBC-MDR y TBC-XDR, ya que el bajo grado de calidad de la evidencia sobre su potencial utilidad sólo permite hacer recomendaciones condicionales. También se están estudiando esquemas que prescindan del empleo de fármacos inyectables, como la kanamicina, con el fin de mejorar la tolerancia y adherencia de los tratamientos y reducir su toxicidad ${ }^{11-12}$.

Esta situación motivó que se estableciera un encuentro de especialistas a través de vídeo conferencias entre Estados Unidos y Sudáfrica para evaluar las metodologías de investigación y protocolos de estas nuevas terapias y las eviden- cias sobre su eficacia. Las conclusiones fueron publicadas recientemente ${ }^{13}$.

Repasaremos brevemente lo que se conoce de los nuevos fármacos que ya están disponibles $\mathrm{y}$ de otros ya conocidos, pero con nuevas indicaciones. De sus diferentes asociaciones se espera que deriven esquemas cada vez más cortos y eficaces $^{14-16}$.

\section{Nuevos fármacos disponibles}

1. Bedaquilina $(B D Q)$ : su nombre comercial es SIRTURO del laboratorio Janssen de Bélgica. Actúa interfiriendo con las fuentes de energía intracelular del bacilo tuberculoso. Su uso contribuye a lograr una conversión más rápida del cultivo cuando se agrega a los esquemas actuales de TBC-MDR. Se debe tener precaución con su asociación con rifampicina ya que ésta al inducir la unidad CyP3A4 del citocromo P450 puede reducir los niveles plasmáticos de BDQ. También al combinarla con delamanid, moxifloxacino o clofazimina puede acentuar sus efectos cardiovasculares con prolongación del intervalo QT en el electrocardiograma. Puede presentar toxicidad hepática en 6-8\% y en algunas oportunidades determina náuseas, vómitos y artralgias. El máximo nivel plasmático se alcanza entre 4 y $6 \mathrm{~h}$ después de su ingesta; se distribuye en todos los tejidos. $\mathrm{Su}$ vida media es prolongada (24-30 h).

La BDQ es metabolizada en el hígado produciendo metabolitos menos activos y más tóxicos. Se acumula dentro de los macrófagos, lo que permite su acción sobre los bacilos intracelulares, pero tiene una menor penetración a los focos caseosos pulmonares. Puede asociarse a fluoroquinolonas y linezolid, pero hay que considerar que tiene resistencia cruzada con la clofazimina. Las dosis habituales son de $400 \mathrm{mg}$ diarios durante 2 semanas seguidas de $200 \mathrm{mg}$ cada $48 \mathrm{~h}$ durante 2 a 6 meses $^{17-18}$.

2. Delamanid (DLM, OPC-67683): ha sido desarrollado por el laboratorio Otsuka. Es una pro-droga que actúa inhibiendo la síntesis de ácidos micólicos (acción bactericida) en bacilos en activa multiplicación. También tiene actividad sobre bacilos latentes intracelulares, con una acción esterilizante similar a la de la rifampicina. No tiene actividad sobre otras bacterias. Es notable su efecto en reducir la mortalidad de los pacientes, logrando la conversión precoz del cultivo en 2 meses. A pesar de aumentar el intervalo QT del ECG, no produce una mayor proporción de efectos cardiovasculares, aunque al asociarlo a fluoroquinolonas o clofazimina el intervalo QT 
puede verse más afectado. Tiene una baja toxicidad hepática ya que no se metaboliza mediante el citocromo P450. Su vida media en plasma es de 30 a $38 \mathrm{~h}$. Su uso por 6 meses o más aumenta su eficacia. Los ensayos en curso demuestran curación de $55 \%$ cuando se asocia durante 2 meses a los esquemas actuales de TBC-MDR; pero, si se utiliza durante 6 meses la curación alcanza a $74,5 \%$ y la letalidad se reduce a $2,9 \%$. Actualmente se ha extendido su indicación a niños y pacientes con VIH. La dosis habitual es de 100 mg cada $12 \mathrm{~h}^{19,20}$.

3. Pretomanid (PA-824): este fármaco forma parte del desarrollo de terapias apoyadas por la "Alianza Global para el Desarrollo de Drogas Antituberculosas". Estas terapias han sido diseñadas para el uso de dosis únicas diarias por vía oral de fácil aplicación y de precio abordable. Estos esquemas han sido utilizados para el rescate de pacientes con TBC-MDR en Sudáfrica, asociando bedaquilina, linezolid y protiomanid durante 4 a 6 meses. También se está ensayando en la TBC sensible para reducir el tiempo de tratamiento ${ }^{21}$.

4. Fluoroquinolonas $(F Q N)$ : tienen una muy buena actividad bactericida, similar a la isoniacida, pero poca acción esterilizante. Estos fármacos pueden penetrar en las lesiones cavitarias y en los granulomas. Existen cepas resistentes iniciales debido a mutaciones de la ADN gyrasa secundarias al uso indiscriminado del fármaco para el tratamiento de infecciones respiratorias en casos de TBC no diagnosticadas. Las FQN más utilizadas son el levofloxacino y el moxifloxacino. Las dosis más utilizadas de moxifloxacino varían entre 400 y $800 \mathrm{mg}$ diarios por vía oral. Los efectos adversos más conocidos son la prolongación del intervalo QT y la tendinitis. Estos fármacos también están siendo indicados en la quimioprofilaxis de contactos de TBC-MDR en niños y adultos en algunos países como Vietnam y Sudáfrica ${ }^{22-23}$.

5. Linezolid (LZD): actúa inhibiendo la síntesis proteica mitocondrial. Su toxicidad es dosis dependiente. Con una mayor dosis inicial $(600 \mathrm{mg}$ cada $12 \mathrm{~h}$ durante $48 \mathrm{~h}$ ) tiene una actividad bactericida precoz similar a la de la isoniacida, pero si se prolonga puede producir mielosupresión y neuritis óptica o periférica. El efecto óptimo del fármaco se obtiene con dosis iguales o superiores a $600 \mathrm{mg}$ diarios durante un tiempo prolongado, pero con esta posología son frecuentes las reacciones tóxicas. Con la administración de hasta $600 \mathrm{mg}$ diarios la suspensión del fármaco por efectos adversos puede llegar a 30\%; con dosis superiores a $600 \mathrm{mg}$ diarios estos pueden alcanzar a $61 \%$. Así, se aconseja reducir las dosis desde $600 \mathrm{mg}$ a $300 \mathrm{mg}$ diarios luego de 3 a 4 meses de su empleo con el objetivo de disminuir los efectos adversos. También puede utilizarse en forma trisemanal a dosis de $1.200 \mathrm{mg}$ cada $48 \mathrm{~h}$, luego de la negativización del cultivo, como una forma de reducir la toxicidad. También podría usarse intermitente desde el inicio si el resto del esquema terapéutico es suficientemente robusto en fármacos activos ${ }^{24-25}$.

6. Clofazimina (CFZ): se acumula dentro de los macrófagos generando especies reactivas oxidativas que inhiben la respiración celular de las micobacterias y provocan la apoptosis de los macrófagos. Se concentra en el tejido adiposo y en las células retículo-endoteliales. Tiene una actividad bactericida prolongada y también actúa sobre la población bacilar latente. Su vida media es de 10 días. La dosis habitual es de $100 \mathrm{mg}$ diarios, pero debe modificarse según la función hepática. Entre sus efectos colaterales destacan la pigmentación reversible de la piel, que puede afectar a más del $75 \%$ de los casos, los trastornos gastrointestinales en hasta el $50 \%$ y la prolongación del intervalo QT. Este fármaco puede tener resistencia cruzada con la bedaquilina. Se ha utilizado en terapias acortadas de TBC-MDR como el esquema de Bangladesh con curaciones de hasta $87,5 \%$ y en el protocolo STREAM con éxito en $73,6 \%$ de los casos. Existen ensayos en curso que permitirán definir con más exactitud su eficacia, sus dosis óptimas, los efectos colaterales y su aplicación en pacientes con $\mathrm{VIH}^{26-30}$.

\section{Conclusiones}

Los mejores diseños de investigación y la mayor cobertura de los tratamientos de la TBC$\mathrm{MDR}$, asociados a los nuevos fármacos, producirán a corto plazo esquemas más breves, más eficaces y menos tóxicos para los pacientes, ya que con las terapias actuales sólo $23 \%$ de los casos de TBC-MDR logran iniciar una terapia de segunda línea. La generación de nuevos medicamentos es un proceso que a la industria le demanda casi una década y es difícil que podamos contar con ellos en estos momentos. Sin embargo, debemos estar preparados para su pronta introducción en amplia escala a nivel mundial.

\section{Bibliografía}

1.- Companion handbook to the WHO guidelines for the programmatic management of drug-resistant tuberculosis. WHO. 2014.

2.- JOHNSTON J C, SHAHIDI N C, SADATSAFAVI M, 
FITZGERALD J M. Tuberculosis: A Systematic Review and Meta-Analysis. PLoS One 2009; 4 (9): e6914. doi: 10.1371/journal.pone.0006914.

3.- AHUJA S D, ASHKIN D, AVENDANO M, BANERJEE R, BAUER M, BAYONA J N et al. Multidrug Resistant Pulmonary Tuberculosis Treatment Regimens and Patient Outcomes: An Individual Patient Data Meta-analysis of 9,153 Patients. PLoS Medicine, 2012, 9 (8), e1001300. http://doi.org/10.1371/journal. pmed. 1001300 .

4.- ORENSTEIN E W, BASU S, SHAH N S, ANDREWS J R, FRIEDLAND G H, MOLL A P et al. Treatment outcome among patients with multidrug-resistant tuberculosis: systematic review and meta-analysis. Lancet Infect Dis 2009; 9: 153-61.

5.- CEGIELSKI J P, DALTON T, YAGUI M, WATTANAAMORNKIET W, VOLCHENKOV G V, VIA L E et al. Extensive drug resistance acquired during treatment of multidrug-resistant tuberculosis. Clin Infect Dis 2014; 59: 1049-63.

6.- COX H S, SIBILIA K, FEUERRIEGEL S, KALON S, POLONSKY J, KHAMRAEV A K et al. Emergence of extensive drug resistance during treatment for multidrug-resistant tuberculosis. New Engl J Med 2008; 359: 2398-400.

7.- SHIN S S, KESHAVJEE S, GELMANOVA I Y, ATWOOD S, FRANKE M F, MISHUSTIN S P et al. Development of extensively drug-resistant tuberculosis during multidrug-resistant tuberculosis treatment. Am J Respir Crit Care Med 2010; 182: 426-32.

8.- WORLD HEALTH ORGANIZATION. Global Tuberculosis report 2015. WHO/HTM/TB/2015.22. Geneva. Switzerland: WHO, 2015.

9.- VAN DEUN A, MAUG A K, SALIM M A, DAS P K, SARKER M R, DARU P et al. Short, highly effective, and inexpensive standardized treatment of multidrugresistant tuberculosis. Am J Respir Crit Care Med 2010; 182: 684-92. Doi:10.1164/rccm. 201001-0077OC. Epub 2010 May4.

10.- AUNG K J, VAN DEUN A, DECLERCQ E, SARKER M R, DAS P K, HOSSAIN M A et al. Successful 9-month Bangladesh regimen for multidrug-resistant tuberculosis among over 500 consecutive patients. Int $\mathrm{J}$ Tuberc Lung Dis 2014; 18:1180-7.

11.- GUALANO G, CAPONE S, MATTEELLI A, PALMIERI F. New antituberculosis drugs: from clinical trial to programmatic use Infect Dis Rep. 2016; 8 (6569): 4349. doi: 10.4081/idr.2016.6569.

12.- DR-TB Clinical Trials Progress Report. RESIST-TB. Last updated june 24, 2017.

13.- HORSBURGH J R CR, RUSEN I D, MITNICK C D. Global MDR-TB clinical trials Landscape Meeting. Int J Tuberc Lung Dis 2016; 20: S 1-57.

14.- WHO. Treatment guidelines for drug-resistant Tuberculosis 2016 update October 2016 revision.

15.- SULLIVAN T, BEN AMOR Y. Global Introduction of
New Multidrug-Resistant Tuberculosis Drugs-Balancing Regulation with Urgent Patients Needs. Emerg Infect Dis 2016; 22 (3). doi: 10.3201/eid2203.151228.

16.- PODANY A T, SWINDELLS S. (2016). Current strategies to treat tuberculosis. F1000Research, 5, F1000 Faculty Rev-2579. http://doi.org/10.12688/f1000research.7403.1.

17.- BENNI J M. Bedaquiline: A promising new agent for the treatment of MDR-TB. International Journal of Drug Development and Research. 2013; 5: 24-8.

18.- The use of Bedaquiline in the treatment of multidrugresistant tuberculosis. Interim policy guidance. WHO, 2013.

19.- GLER M T, SKRIPCONOKA V, SÁNCHEZ-GARAVITO E, XIAO H, CABRERA-RIVERO J L, VARGASVÁSQUEZ D E et al. Delamanid for Multidrug-Resistant Pulmonary tuberculosis. N Engl J Med 2012; 366: 2151-60.

20.- The use of delamanid in the treatment of multidrugresistant tuberculosis. Interim policy guidance, WHO, 2014.

21.- SLOAN D J, LEWIS J M. Management of multidrugresistant TB: novel treatments and their expansion to low resource setting. Trans R Soc Trop Med Hyg 2016; 110: 163-72.

22.- YEW W W, LANGE C, LEUNG C C. Treatment of tuberculosis: update 2010 Eur Respir J 2011; 37: 441-62.

23.- ZIGANSHINA L E, SQUIRE S B. Fluorquinolones for treating tuberculosis (review). The Cochrane Collaboration, 2009.

24.- XU H B, JIANG R H, LI L, XIAO H P. Linezolid in the treatment of MDR-TB: a retrospective clinical study. Int J Tuberc Lung Dis 2012; 16: 358-63.

25.- SOTGIU G, CENTIS R, D'AMBROSIO L, ALFFENAAR J W, ANGER H A, CAMINERO J A et al. Efficacy, safety and tolerability of linezolid containing regimens in treating MDR-TB and XDR-TB: Systematic review and meta-analysis. Eur Respir J 2012; 40: 1430-42.

26.- DALCOLMO M, GAYOSO R, SOTGIU G, D’AMBROSIO L, ROCHA J L, BORGA L et al. Effectiveness and safety of clofazimine in multidrug-resistant tuberculosis: a nationwide report from Brazil. Eur Respir J 2017, 49: 1602445 doi: 10.1183/13993003.024452016.

27.- TANG S, YAO L, HAO X, LIU Y, ZENG L, LIU G et al. Clofazimine for the treatment of multidrug-resistant tuberculosis. Prospective, Multicenter, randomized controlled Study in Chine. Clin Infect Dis 2015 ,60:1361-7. doi: 10.1093/cid/civ027.

28.- MOODLEY R, GODEC T R. On behalf of the STREAM Trial Team. Short-course treatment for multidrug-resistant tuberculosis: the STREAM Trials. Eur Respir Rev 2016; 25: 29-35.

29.- NUN A J, RUSEN I D, VAN DEUN A, TORREA G, PHILLIPS P P J, CHEN-YUAN CHIANG et al. Evaluation of a Standardized Treatment regimen of anti- 
tuberculosis drugs for patients with multi-drug-resistant Tuberculosis (STREAM): Study protocol for a randomized controlled Trial. Trials 2014 doi:10.1186/17456215-15-353 Biomed Central Trials.

30.- PELOQUIN C A. Clofazimine Pharmacology and usage details wwww.medicineindia.org. Infectious disease pharmacokinetics laboratory. College of Pharmacy and the Emerging Pathogens Institute. University of Florida. wwww.resisttb.org/wp/clinical-pharmacology of clofazimine.pd.

\section{Correspondencia a:}

Dr. Victorino Farga C.

Providencia 2608 Piso 3. Santiago, Chile.

Email: vfarga@gmail.com 